\title{
Prävention ist Ärztesache - transparente Kommunikation ist ihre Grundlage
}

\author{
Das Versagen der Präventionsbemühungen der letzten Jahre kann nicht nur der PR- \\ und Marketingmaschinerie der Gegner zugeschrieben werden. Der schweizerische \\ Gesundheitssektor selbst hat zur schwierigen Situation der Prävention beigetragen.
}

Strukturelle (d.h. gesetzliche) Massnahmen der Prävention sind wirksam: Auf Volksgesundheit und Kosten wirken sie sich positiv aus. Der Gesundheitssektor hatte sich für das Präventionsgesetz und für die Volksinitiative «Schutz vor Passivrauchen» ausgesprochen. Beide Vorlagen, welche die strukturellen Public-Health-Massnahmen der nächsten Jahrzehnte hätten bilden müssen, wurden jedoch von Parlament und Volk abgelehnt. Offenbar gelingt den Präventionsargumenten der Durchbruch in der Schweiz nicht. Dieser Artikel skizziert die Arbeit der Präventionsgegner, um danach diejenige der Gesundheitsorganisationen und der Ärzte zu beleuchten.

\section{Die Öffentlichkeitsarbeit der Gegner}

Das Volk lehnte 1992 mit 75\% der Stimmen die Zwillingsinitiative $\mathrm{ab}$, die das für audiovisuelle Medien bestehende Reklameverbot für Alkohol und Tabak hätte ausdehnen sollen. Die damalige PR-Kampagne lässt auf die prinzipielle Strategie der Gegner schliessen. Erstens: die dem Volk vorgelegte konkrete Frage zur Grundsatzdebatte deformieren. Damals: «Ja oder Nein zu einem Allgemeinen Reklameverbot in einer offenen Gesellschaft?»; im Jahre 2012: «Ja oder Nein zum Absoluten Rauchverbot?» Zweitens: Präventionsbefürworter isolieren/verunglimpfen. Drittens: die allgemeine Information der Öffentlichkeit über strukturelle Gesundheitsprävention lückenhaft halten. Der Ausgang der Volksabstimmungen beweist die erfolgreichen PR- und Marketingmassnahmen.

\section{Abhängigkeit der Medien}

Die Abhängigkeit der Medien [1]* von der Industrie erklärt einseitige Information des Publikums und dadurch den Einfluss auf den demokratischen Entscheidungsprozess [2]. Die Arbeitsgruppe Tabakprävention (AT) analysierte die Printmedien in den vier Monaten vor Abschluss der Unterschriftensammlung der Lungenliga und fand auf total 999 Beiträgen nur 91 für den Passivrauchschutz, 263 dagegen, die restlichen neutral [3]. Es überwogen Skepsis, extreme Standpunkte, und Unkenntnis des Themas. Auch angesehene Medien arbeiteten tendenziös; die
NZZ am Sonntag erklärte am 19. August 2008: «Nein, das Thema Rauchverbot im öffentlichen Raum wird von uns momentan nicht weiter verfolgt», und $L e$ Temps schwieg über die Passivrauchdebatte seit 2008, publizierte aber während der Abstimmungskampagne im September 2012 den Hintergrundartikel «Diese Laster, die man ausrotten möchte» [4], was perfekt in die Strategie der Gegner passte, welche die Initianten als Moralisten verunglimpft.

Von Medien systematisch wiederholte Vorurteile verankern sich bei lückenhafter Information besser. So «Gesundheitstaliban/-ayatollah», die ertönten, sobald von Prävention die Rede war. Auch in der Zeitschrift der Schweizer Spitäler Competence zu finden [5], wurden die Begriffe erstmals in der Gewerbezeitung vom April 2008 verwendet [6], und in der Sendung Zehn vor zehn und im Blick lautstark wiederholt. Der Zweck war, Prof. Thomas Zeltner zu verunglimpfen. Die Zielscheibe war kein Zufall. Einerseits war Zeltner der seit Jahren angesehenste Direktor des Bundesamtes für Gesundheit, der auch in der Tabakprävention der WHO erfolgreich war [7]; andererseits war er der hochrangige Staatsangestellte [8], der öffentlich den Filz zwischen Industrie und Politik für den schleppenden Fortschritt der strukturellen Prävention in der Schweiz verantwortlich zu machen wagte [9].

Andere hieben in die gleiche Kerbe.Wie Beda Stadler, der in Weltwoche und NZZ Prävention als Gebaren von Eiferern darstellte [10]. Der wirksamste Beitrag dieses Immunologen der Universität Bern war «Enttarnte Wissenschaft» [11]. Der Text disqualifizierte die Studien über den Rückgang der Koronarsyndrome nach der Einführung von Passivrauchregelungen als Erfindungen. Er erschien, wie zufällig, im Sommer 2008 und diente der Desinformation der Parlamentier, die im folgenden Herbst die Gesetzesvorlage zum Passivrauchschutz wieder aufnehmen sollten [12].

\section{Gesetzgeber}

Die Gesetzgeber waren dem Druck von Economiesuisse ausgesetzt, welche die Tabakmultis sogar in 


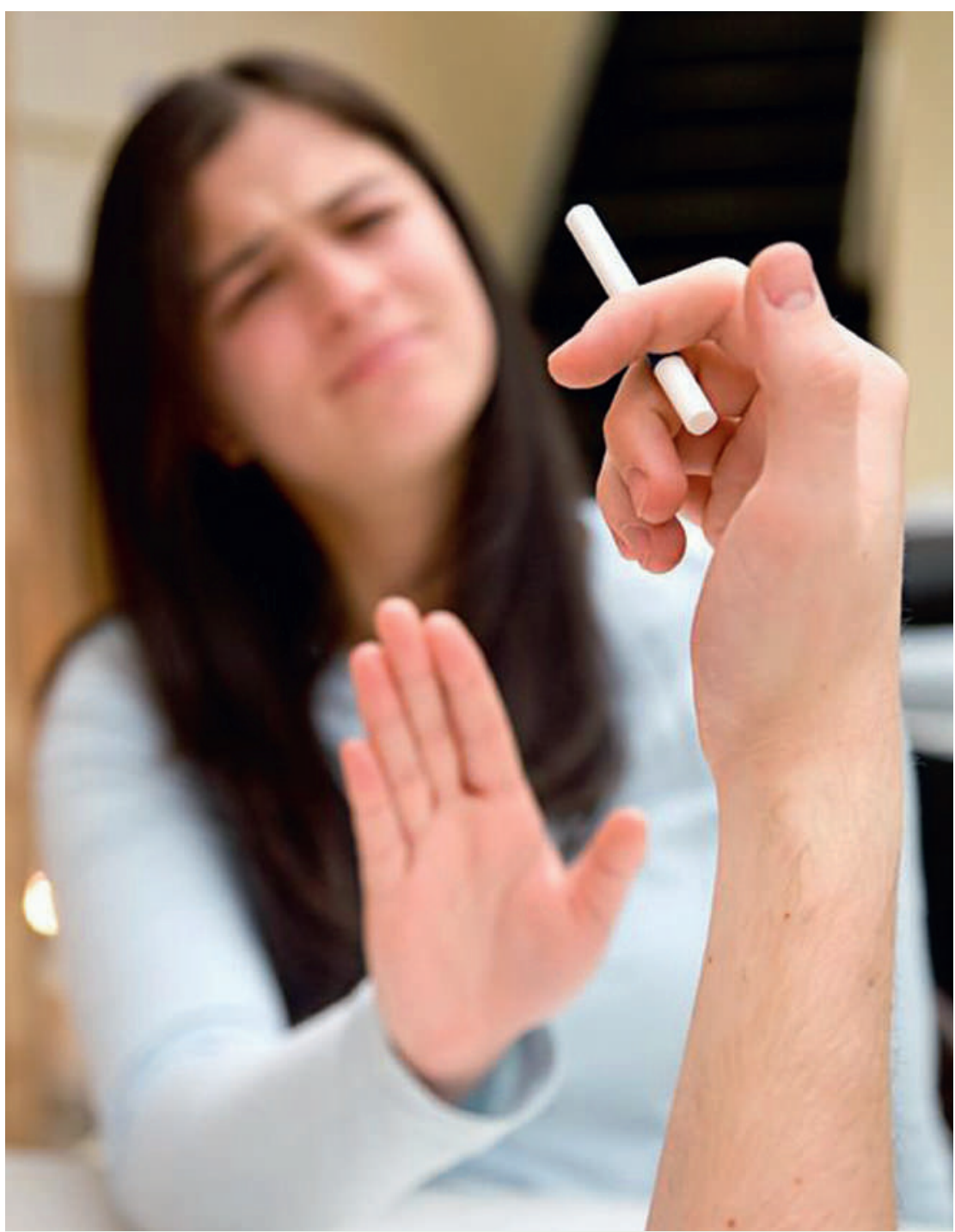

Warum gibt es immer noch Widerstand gegen umfassenden Schutz gegen Passivrauchen?

Australien vertritt [13]. Der Gewerbeverband gründete 2008 die Allianz der Wirtschaft für eine massvolle Prävention, in der die Zigarettenindustrie und Economiesuisse vertreten sind, die von der CVP und der SVP unterstützt wird (www.awmp.ch), und welche die Tabakmultis als Drahtzieher zu tarnen vermochte [14]. Ihr Einfluss auf die Politiker zwischen der äusserst knappen Annahme des «Gastro-boroAlibi»-Bundesgesetzes 2008 und der parlamentarischen Debatte der Initiative ab 2010, dürfte noch zugenommmen haben. Denn mit der im Nationalrat im Dezember 2011 ausgebreiteten Argumentation erscheint die Ablehnung der Initiative mit 118/55 Stimmen nicht als plausibel [15]. Auch die Erklärung des Bunderates [16] in dieser Debatte vor dem Nationalrat kann man unter diesem Aspekt betrachten, da der Erläuterungstext zur Volksabstimmung Verwirrung stiftete: Didier Burkhalter unterstellte, die Abnahme der Hospitalisierungen von Koronarsyndro- men und Bronchitisexacerbationenen sei dem Bundesgesetz von 2008 zuzuschreiben und die Initiative sei «extrem», da sie das Rauchen am Einzelarbeitsplatz verbiete. Diese offiziellen Unwahrheiten [17] wurden von seinem Nachfolger Berset und von den Medien wieder aufgenommen, ohne je richtiggestellt zu werden [18].

Präventionsgegner stützten sich also auf breite Kreise der Wirtschaft und deren Verbindungen zu Medien, Politikern und Öffentlichkeit, um die Initiative «Schutz vor Passivrauchen» zu torpedieren.

\section{Die Allianz der Gesundheits-}

\section{organisationen war nicht solide.}

\section{Die Arbeit der Gesundheitsorganisationen}

Trotz der ungleich verteilten Mittel war die Ausgangslage der Prävention nicht verloren; das Publikum war positiv eingestellt [19], und ihre wissenschaftliche Verankerung und deren Nutzen sind unbestritten. Gesundheitsberufe zu sensibilisieren, war also möglich. Als die Initiative 2009 lanciert wurde, bildete sich eine Allianz von Gesundheitsorganisationen, die Unterstützung für die Abstimmung versprach. Da viele Allianzpartner als Vereine organisiert sind, konnten interne Kommunikationsmittel verwendet werden, um Mitglieder, Angestellte, Sympathisanten, Spender und Publikum zu informieren.

Es erwies sich aber, dass die Botschaften der Lungenliga nur fragmentarisch verbreitet wurden. Selbst während der Abstimmungskampagne forderten nicht alle Lungenligen die Besucher ihrer Internetportale auf, im September 2012 für die Initiative zu stimmen. Mit wenigen Ausnahmen wies keines der E-Portale der Krebsligen auf die Volksabstimmung hin, und dies galt auch für andere Partner. Dies bestätigte, dass die Allianz der Gesundheitsorganisationen nicht solide war. Es wurde früh klar, dass einige kantonale Lungenligen sich weder an der Unterschriftensammlung beteiligen noch sich öffentlich positionieren würden. Die nationale Initiative wurde so beispielsweise von der Lungenliga Waadt durch Komiteebeschluss spät, erst kurz vor Abschluss der Unterschriftensammlung, unterstützt.

\section{Die Arbeitsgemeinschaft Tabakprävention (AT)}

Im März 2012 entpuppte sich die breite Allianz als Papiertiger: Nach Debatte in Bundesrat und Parlament stellte die Bundeskanzlei formell die Frage an die Initianten, ob die Initiative aufrechterhalten werde. Überraschend plädierte die Vertreterin der AT für ihren Rückzug! Eine Argumentation ihres Komitees [20] stützte das Votum. Als Motiv wurde die befürchtete Abstimmungsniederlage genannt; der 
* Anm. d. Red.: Am Anlass wurde von der Moderatorin und von der Präsidentin von Public Health Schweiz auf diesen Umstand hingewiesen.
Rückzug wurde dagegen als «staatspolitisch vernünftiger Akt eingestuft, der weiteren Anliegen Goodwill verschafft». Und weiter : «(...) das Präventionsgesetz hat zurzeit Chancen, vom Parlament angenommen zu werden (...) Festhalten an der Initiative wird als Zwängerei interpretiert und kann das Scheitern des Präventionsgesetzes bedeuten.» Diese Aussagen sind nicht leicht zu deuten. Unter anderen Hypothesen könnte eine Absprache zwischen den Lagern erwogen werden.

\section{Die Ärzteschaft}

In der Präventionsallianz fehlten anfänglich die wichtige FMH und die Schweizerische Gesellschaft für Innere Medizin (SGIM), die grösste Schweizer Ärztegesellschaft. Die Dachorganisation der Ärzte stellte sich erst Ende 2009 durch Beschluss ihrer Delegierten hinter die Initiative. Die FMH argumentiert noch am 1.4.2009, dass die «Initiative Kräfte binden (könnte), (...) die im Kampf um das neue Präventionsgesetz gebraucht würden». Die SGIM konnte sich im Jahre 2008 während der Parlamentsdebatte um das «Gastro-boro-Alibi»-Gesetz nicht positionieren, wobei auch Sponsoren erwähnt wurden [21]. Nach Vorstössen von Mitgliedern beschloss die Generalversammlung der SGIM 2011 jedoch, die Volksinitiative zu unterstützen. Das Traktandum kam ohne Empfehlung des Komitees zur Abstimmung, und entgegen dem Beschluss der Generalversammlung hat die SGIM sich nie öffentlich zur Initiative positioniert.

Für andere Ärzteorganisationen gilt dasselbe. Auf die Einladung an die Organisatoren des Jahreskongresses «Swiss Public Health» Ende August in Lausanne, im Hinblick auf die Abstimmung im September eine Pressekonferenz zu organisieren, wurde geantwortet, das Programm sei schon abgeschlossen Die Präsidentin präzisierte am 12.9.2012 in einer EMail-Nachricht an den Autor dieses Artikels, dass «sich Public Health Schweiz (...) neben dem Präventionsgesetz nicht noch für eine zweite Gesetzesvorlage mit gleicher Kraft engagieren kann». Selbst bei Ärztegesellschaften, die von Anfang an der Allianz angehörten, war die Antwort: «Die Mitglieder des Organisationskomitees erachten den Anlass des Fortbildungskongresses als ungeeignet für eine öffentliche Positionierung der Schweizer Ärzte» (Kongress «family docs», 26.7.2012).

\section{Ärztezeitschriften}

Ärztezeitschriften verhielten sich diskret: Die Redaktion der Schweizerischen Ärztezeitung SÄZ organisierte mit der Berner Ärztegesellschaft am 6. Juli 2011 die Podiumsdiskussion «Prävention zwischen sinnvoller Steuerung und Bevormundung». Weder in der Ankündigung noch in der Berichterstattung der $S \ddot{A} Z$ danach [22], wurde dem Publikum bezw. der Leserschaft erklärt, dass der am Podiumsgespräch teilnehmende Hans Ulrich Bigler zwar als Direktor des
Schweizerischen Gewerbeverbandes die KMUs vertritt, aber auch Schriftführer der Allianz der Wirtschaft für eine massvolle Präventionspolitik ist.* Eine Allianz, der die bekanntlich mit Philip Morris verbundene Gastrosuisse [23], Economiesuisse und die Tabakmultis der Schweiz angehören [14]! - Eloquent das Schweigen der Experten der Revue médicale Suisse: Ihre Ausgabe vom 11.7.2012 widmete sie ganz der Prävention, ging im Editorial [24] sowie in einem ausführlichen Artikel auf strukturelle Tabakprävention [25] und auf die Tabakrahmenkonvention der WHO ein; aber in der ganzen Ausgabe ist kein Wort zur Passivrauchinitiative noch zur Volksabstimmung vom 23.9.2012 zu finden! - Eine Aufforderung zur Stimmabgabe fand sich weder in der Patientenzeitung Sprechstunde noch in ihrer französischsprachigen Schwester Planète Santé.

\section{Kommentar}

Das Versagen der Präventionsbemühungen der letzten Jahre der PR- und Marketingmaschinerie der Gegner allein zuzuschreiben, ist nicht plausibel. Der schweizerische Gesundheitssektor selbst hat wesentlich zur schwierigen Situation der Prävention beigetragen. Dass ein Abstimmungsmisserfolg Motiv sein sollte, die Initiative zurückzuziehen, kann weder öffentlich noch vor Allianzpartnern vertreten werden. Denn das bedeutet, dass man am eigenen Standpunkt zweifelt. Das Argument erstaunt umso mehr im Munde der AT, die regelmässig über die Tabakindustrie berichtet, die strukturelle Tabakprävention weltweit mit diskutablen Manövern zu verhindern sucht. Wenn die AT überzeugt war, dass das «Gastro-boro-Alibi»-Bundesgesetz zu akzeptieren sei, dann hätte sie nicht dem Initiativkomitee beitreten dürfen. Der Mangel an Transparenz ist offensichtlich.

Die FMH, die AT und Public Health Schweiz argumentierten, dass man sich nicht gleichzeitig für das Präventiongesetz und für die Initiative einsetzen könne. Diese Sichtweise zeugt von einer groben Fehleinschätzung des politischen Kräftespiels. Hier gelten allein Stimmenzahl und Druck der öffentlichen Meinung: Je mehr der Gesundheitssektor für eine konkrete Frage der Prävention jenseits von Parteigrenzen die Bevölkerung mobilisieren kann, desto weniger können die Parlamentarier auf die Industrielobby hören. Kurzum: Wäre der Kuhhandel «Rückzug der Initiative gegen Präventionsgesetz» als Weg vorgezeichnet, beide Vorlagen bachab zu schicken? Der Rückzug der Initiative hätte jedenfalls der Tabakindustrie einen Vorteil verschaft. Sie hatte eine analoge Strategie schon erfolgreich in Kalifornien verfolgt [26]. Man entdeckt perplex, dass im Vorstand der Arbeitsgemeinschaft Tabakprävention, zum Rückzug neigend, sowohl die FMH, die Krebsliga als auch die Lungenliga Schweiz [27] vertreten sind, Allianzpartner, welche die Plakate der Initiative zeichneten. 


\section{Prävention ist Ärztesache [28]}

Seit es Ärzte gibt, werden sie von Patienten als Experten gesunder Lebensweise konsultiert. Ihr Schweigen in dieser öffentlichen Debatte kommt aktiver Desinformation gleich: Von wem sollten die Stimmbürger sachliche Information erhalten, wenn nicht von ihren Ärzten?

Statuten der FMH, der Kantonalen Ärztegesellschaften, Charten der AT, der Spitäler der Schweiz, von Public Health Schweiz u. a. verankern Förderung der Volksgesundheit und Information der Öffentlichkeit als ärztlichen Auftrag. Die Expertenrolle der Ärzte für die öffentliche Gesundheit wird von Berufsorganisationen [29] und von der WHO festgehalten [30]. Was muss man davon halten, wenn ein Präventivmediziner erklärt, sich nicht öffentlich in der Passivrauchdebattte positionieren zu können, weil sein Universitätsinstitut von Steuergeldern abhängig sei [31]? Ist er doch von der Öffentlichkeit beauftragt, zur Information der Entscheidungsträger von Partikularinteressen unabhängige Wissenschaft zu betreiben!

\section{Schlussfolgerung}

Wenn im wirtschaftlich dominierten Umfeld Gesundheitsorganisationen zur Prävention sich nicht eindeutig öffentlich positionieren, überlassen sie die Entscheidungen Kreisen, die ihre Partikularinteressen weit vor das Gesamtwohl stellen. Die Tabakindustrie lässt der Prävention im politischen Kräftespiel durch Kompromisse, interne Abmachungen, u. ä. keine Chance. Die Rahmenkonvention der WHO hält unmissverständlich fest, dass die Tabakindustrie intergrierter Bestandteil des von ihr verursachten weltweiten Public-Health-Problems ist. Deswegen müssen ihre Verbindungen zu Medien, Politikern, der Zivilgesellschaft und Ärzten in die öffentlich zu führende Debatte einbezogen werden.

Abhängige Medien interessieren sich für solche Zusammenhänge nicht. Die Bevölkerung zu informieren, fällt daher den Gesundheitsorganisationen zu. Ihre Glaubwürdigkeit hängt davon ab, dass ihre Strukturen für Mitglieder und Öffentlichkeit transparent und demokratisch funktionieren. Und nicht in den Verdacht geraten, von der Industrie unterwandert zu sein, wie dies in den Verbänden des Gastgewerbes geschah [23]. Deswegen verlangen wissenschaftliche Gesellschaften und ihre Publikationsorgane weltweit von ihren Verantwortlichen Erklärungen über Interessenkonflikte gegenüber Pharmafirmen und der Tabakindustrie. Angesichts der in der Schweiz beheimateten multinationalen Firmen ist dies eine mehr als legitime Forderung an Gesundheitsorganisationen, Ärztegesellschaften eingeschlossen.

Ärzteorganisationen wären gut beraten, in der Öffentlichkeit gut wahrnehmbar so aufzutreten, wie sich der Praktiker täglich in seiner Konsultation verhält: als unabhängiger, ehrlicher und uneigennütziger Experte in Gesundheitsfragen, der Desinformation entlarvt. Denn das Vertrauen, das uns Patienten und Bevölkerung entgegenbringen, das für den Erfolg unserer Arbeit notwendig ist und unser Ansehen bei Kranken und Angehörigen begründet, hängt wesentlich von unserer Glaubwürdigkeit und unserer Unabhängigkeit ab.

\section{Mitunterzeichner:}

Dr Yves Beyeler, médecine interne, ancien président du Cipret Genève, Genève

Prof. Dr. med. Martin Brutsche, Chefarzt Pneumologie, Kantonsspital St.Gallen

Prof. em. Jean Claude Chevrolet, ancien médecin chef des soins intensifs des HUG, Genève

Dr Jean Georges Frey, Médecine interne et Pneumologie, médecin chef, Centre valaisan de Pneumologie, Montana

Prof. Dr. med. Jürg Hammer, Leitender Arzt und Stellvertretender Chefarzt Pädiatrie, Pneumologie und Intensivmedizin, Universitätskinderspital beider Basel.

Dr . med. Karl Klingler, Pneumologie, Innere Medizin und Schlafmedizin, Lungenzentrum Hirslanden, Zürich

Dr. med. Bruno Knöpfli-Balmer, Pädiatrie, Pneumologie und Sportmedizin, Davos

Dr. med. Alexander Möller, Privatdozent, Leiter Pneumologie, Universitätskinderkliniken Zürich

Dr Reto Olgiati, Pneumologie, Chargé de cours, Université de Fribourg, Délémont

Dr. med. Helmut Oswald, Kinder- und Jugendmedizin, speziell Lungenkrankheiten, Winterthur

Prof. Dr. med. Ralph Alexander Schmid, Präsident der Schweizerischen Gesellschaft für Chirurgie, Past-Präsident der Schweizerischen Gesellschaft für Thoraxchirurgie, Direktor Universitätsklinik für Thoraxchirurgie, Inselspital Bern

Dr Hubert S. Varonier, PD, Pédiatrie et Allergologie, Président d'honneur des Cipret Genève et Valais, Crans-Montana

\section{Interaktiver Artikel}

Wollen Sie diesen Artikel kommentieren? Nutzen Sie dafür die Kommentarfunktion in der OnlineVersion oder sehen Sie nach, was Ihre Kolleginnen und Kollegen bereits geschrieben haben: www.saez.ch/aktuelle-ausgabe/interaktive-beitraege/ 


\section{Literatur}

1 Daniel Eskenazi: PubliGroup n'exclut pas une restructuration. Le numéro un suisse souffre du recul des annonces dans la presse écrite en 2012. Le Temps 10 mars 2012

2 Kurt Imhof: Oui la Presse décline et la démocratie en souffre. Le Temps, 16.11.2010.

3 T. Beutler: Medienberichte zum Schutz vor Passivrauchen. AT information, Sommer 2010.

4 Emmanuel Garessus: Ces vices qu'on aimerait éradiquer. Le temps, 4 septembre 2012.

5 Simplicissimus: Der abgehackte Mahn- und Drohfinger. Compétence H+ Forum, 11/2012.

6 Patrick Lucca in Schweizerische Gewerbezeitung. April 2008.

7 Zeltner T et al.: Tobacco Companies Strategies to undermine Tobacco Control Activities of the WHO. Report of the Committee of Experts on Tobacco Industry Documents. WHO/OMS, Geneve, 2000

8 R. M. Kälin: Diener des Staates ... Schweiz Ärztezeitung. 2010;91(17):690-91.

9 Pierre Hazan: Le patron de la santé publique dénonce la collusion entre politiques et Cigarettiers. Le Temps, 21.10.1998.

10 Beda Stadler: Vom Schaden staatlicher Prävention. Weltwoche 9.3.2008. Wie gefährlich ist Passivrauchen? Weltwoche, 18.1.2007. Als Nächstes wird der Handel mit Kohlenhydraten reguliert. NZZ am Sonntag, 14.8.2011.

11 Beda Stadler: Enttarnte Wissenschaft, Weltwoche, 32/8; 7.8.2008. Im Sommer 2008 erschien Pell JP et al.: Smoke free legislation and Hospitalizations for Acute Coronary Syndrome, N.E.J. M. 2008: 359:5,482-491, eine sauber ausgeführte Studie, welche den Rückgang der akuten Koronarsyndrome nach Einführung des Rauchverbotes im öffentlichen Raum in Schottland dokumentierte. Diese Ergebnisse wurden in der Schweiz bestätigt, durch Bonetti et al.: Incidence of acute myocardial infarction after implementation of a public smoking ban in Graubünden, Switzerland. Two year follow-up. Swiss Med. Wkly, 2011; 141: w13206. www.smw.ch. Beda Stadler disqualifizierte die Schweizer Studie prompt mit dem Artikel «Es ist ein Wunder», Weltwoche, 14.1.2010.

12 R. M. Kälin: Prävention und Glaubwürdigkeit von Gesundheitspolitikern: ein Minenfeld. Schweiz Ärztezeitung. 2012; 93(23):873-878.

13 Economiesuisse, Thomas Pletscher: Consultation on the exposure draft Tobacco Plain Packaging Bill 2011 and Consultation Paper, to Department of Health and Ageing, Canberra Australia. May 19. 2011

14 Die Allianz der Wirtschaft für eine massvolle Prävention besteht aus folgenden Mitgliedern: Schweizerischer Gewerbeverband, Economiesuisse, ASCO (Verband Konzertlokale, Cabarets, Dancings und Discotheken), Gastrosuisse, Hotelleriesuisse, Schweizer Bauernverband, Casinoverband, SchweizerWerbung, Zigarrenfabrikanten, Tabakwarenhandel, VISCOM (Schweizerischer Verband für visuelle Kommunikation) usw. Unter den unterstützenden Organisationen finden sich die Christliche Volkspartei (CVP), die Schweizerische Volkspartei (SVP) und Swiss Cigarette (Philip Morris international, British American Tobacco international, Japan Tobacco international).
15 Das Gastro-boro-Alibi Bundesgesetz von 2008 wurde im Nationalrat mit 89 Ja gegen 88 Nein angenommen. Im Ständerat wurde die Vorlage gerade nur durch den Stichentscheid seines Präsidenten Christoffel Brändli, SVP (damals Präsident von santésuisse), zum Gesetz erhoben.

16 Pascal Couchepin, im Ständerat 2008: «Die wirtschaftlichen Argumente sind, ehrlich gesagt, nicht seriös. Niemand (kann) bestreiten, dass Passivrauch etwas Negatives ist. Ich lade Sie ein, für eine Lösung zu stimmen, welche nicht freiheitsbeschränkend ist, die ganz einfach gesund ist, und sich auf die Lösung des Bundesrates zu einigen.» Didier Burkhalter, sein Parteikollege und Nachfolger als Gesundheitsminister empfahl dagegen die Ablehnung der Initiative.

1724 Heures, 19.9.2012: Fumée passive «les adversaires de l'initiative mentent». Interview mit Pierre-Yves Maillard, Regierungsrat Waadt.

18 Die von den Initianten veranlasste juristische Beurteilung durch das BAG zum scheinbaren Widerspruch zwischen Initiative und dem Status quo im Erklärungstext zur Abstimmung hält fest, dass die Regelung des Einzelarbeitsplatzes gemäss Initiative in nichts von der bisher geltenden abweicht. Diese Richtigstellung wurde von den Medien nicht verbreitet.

19 Gemäss Meinungsumfrage im Sommer 2012, bevor die Abstimmungskampagne einsetzte, waren knapp 60\% der Befragten bereit, dem Initiativtext «Schutz vor Passivrauchen» zuzustimmen.

20 Vorstand der AT, Bern 20. März 2012: «Ja zum Rückzug der Initiative zum Passivrauchschutz. An die Mitglieder des Initiativkomitees der Initiative zum Passivrauchschutz. .... Mit deutlichem Mehrheitsbeschluss empfiehlt der Vorstand der Arbeitsgemeinschaft Tabakprävention, die Initiative zurückzuziehen. ... die wichtigsten Organisationen der Prävention, die im Vorstand der AT vertreten sind, haben zum Ausdruck gebracht, dass sie den Rückzug der Initiative empfehlen, dies auch so kommunizieren und dementsprechend den allfälligen Abstimmungskampf nicht unterstützen werden.» 4 Seiten, gezeichnet B. M., Präsident.

21 V. B., Präsidentin des SGIM-Briefs vom 3.4.2008: «... Wir haben die Jahresversammlung nicht als rauchfreie Zone deklariert. Ein Grosskongress kann nicht in der letzten Minute einen derartigen Schritt organisieren. Im Vorfeld müssen die entsprechenden Mit-Organisatoren, die Sponsoren, etc. auf diese Neuerung aufmerksam gemacht werden.»

22 Felicitas Witte: Prävention ist geteilte Verantwortung. Schweiz Ärztezeitg. 2011;92(34):1276-1279. Die Autorin, freie Medizinjournalistin, berichtet über das Podiumsgespräch: Die Tabakindustrie wird nicht erwähnt, die Allianz der Wirtschaft für eine massvolle Präventionspolitik und deren Zusammensetzung ebenso wenig.

23 Die Infiltration von Wirtevereinen durch die Tabakindustrie ist seit den 90er Jahren in Kalifornien belegt (Fogels Testimony vor dem Stadtrat von New York: «Es existierte keine Restaurantbesitzervereinigung vor der Rauchfreiheitsverordnung; wir wurden durch die Tabakindustrie organisiert.»). Der Einfluss auf Gastrosuisse und auf die in der Schweiz domizilierte Ho-Re-Ga international durch Philip Morris ist durch Dokumente der Tabakindustrie für die Jahre 1994 und 1995 bewiesen. Siehe Anmerkungen, online 
$\mathrm{zu}$ «Passivrauchgesetze: Die Bayern haben sich gegen die spanische Konfusion entschieden. Schweiz Ärztezeitung. 2010; 91(41):1618-20.

24 F. Paccaud: Editorial. Quelques enjeux de la médecine préventive. Rev.Méd.Suisse, 2012; 8:1435-36.

25 P. Bovet, J. Cornuz, F. Paccaud: Convention-cadre pour la lutte antitabac: un instrument puissant de santé publique. Rev.Méd.Suisse, 2012; 8:1438-41.

26 Paul Kinney, A-K Associates. To Roger Mozingo and Hurst Marshall. Personal and confidential. Sept. 24.1987. http://legacy.library.ucsf.edu/tid/diz44d00/ pdf. 50660 9215. page 3: «The tobacco industry prudently recognized that effort and resources spent in keeping the proposed tax initiative off the ballot had many benefits. If successfull, it would save the industry the many millions that would have to go into the initiative campaign fight. Even if unsuccessfull, the relatively small amount of resources expended on the organizational efforts to keep the initiative off the ballot, would place the industry miles ahead for the general campaign. This unique and innovative approach of actively fighting to keep an initiative off the ballot would only reinforce to the potential anti-tobacco forces that it will always be a long and grueling battle when you take on tobacco. Recognizing that the most effective approach to any such battle is to contain and, if possible, take away potential resources from the proponents, our initial goal was to contain the California Medical Association. ...»

27 Dokument vom 20.3.2012. «Mitglieder des Vorstandes der AT Schweiz: Krebsliga Schweiz, Lungenliga
Schweiz, RADIX, Herzstiftung Schweiz, Sucht Schweiz, Berner Gesundheit, Public Health Schweiz, Züri rauchfrei, Cipret Genève, Cipret Vaud, FMH, pro Aere, Lungenliga St.Gallen.»

28 FMH Prävention. Christine Romann, Barbara Weil Gesundheitsförderung und Prävention: wohin die Reise gehen soll. Schweiz Ärztezeitung. 2011;92(35):1309.

29 Joint committee of American college of chest physicians, American thoracic society, Asia Pacific Society of Respirology, Canadian thoracic society, European respiratory society, International Union against tuberculosis, in Eur. Respir. J, 1995, 8.18081811: Many communities have recognized the social implication of smoking, and have therefore enacted policy and legislation ... such legislative issues are complex and involve balancing rights and privileges of various heterogeneous groups... in addition to their role as citizens, physicians are leaders with regard to any issues affecting public health. Physicians should therefore be aware, that public policy regarding smoking can be an effective instrument of public health, and they have the responsibility to participate effectively in public debate both as individuals and as members of medical organizations.

30 OMS/WHO 2005: le rôle des professionnels de la santé dans la lutte antitabac.

31 F. P., Institut universitaire de médecine sociale et préventive; Séance d'information. En vue de la votation du 23 septembre 2012 sur l'initiative populaire «protection contre le tabagisme passif», 29.8.2012. 